\title{
EDITORIAL
}

\section{EXPERIENCIAS EN LA GENERACIÓN DE MODELOS DE INTERNACIONALIZACIÓN A LA MEDIDA}

La Rectoría de la Universidad de Ciencias Aplicadas y Ambientales U.D.C.A considera que este aporte, tan sustancialmente valioso, se debe comunicar y compartir con los lectores de la Revista U.D.C.A Actualidad E Divulgación Científica, puesto que es un proyecto de análisis y de estudio sobre la Internacionalización de la Universidad que, en su fase determinante y concluyente, fue diseñado por la directora del Departamento de Relaciones Internacionales, Ximena Cardoso Arango, con base en contribuciones de todos los miembros del subsistema académico de la U.D.C.A. Es importante, igualmente mencionar, que este proyecto fue concebido con referentes y experiencias regionales, nacionales e internacionales y de varias Instituciones de Educación Superior, de la ciudad de Bogotá.

Para los universitarios, interesados en nuestra Revista Científica, considero que este documento será de gran utilidad e impacto y los invito para que lleven a cabo un análisis de su contenido y construyan sus propias conclusiones, con miras a fortalecer los procesos de internacionalización, fundamental hoy en día, para la excelencia académica, de todas las Universidades.

Durante el 2009 y el 2010, la Red Colombiana para la Internacionalización de la Educación Superior $\mathrm{RCI}$, el Ministerio de Educación Nacional y la Asociación Colombiana de Universidades ASCUN, implementaron un proyecto para fortalecer la internacionalización de la Educación Superior en Colombia, consistente en la construcción de capacidades y de competencias institucionales y en la generación de modelos de internacionalización "a la medida", en Instituciones de Educación Superior de todo nivel y carácter, con características diferentes de ubicación, de contexto y de condiciones institucionales. El producto final del proyecto Acción para el fortalecimiento de la Internacionalización de las IES Colombianas fue la generación de políticas y de planes estratégicos institucionales de internacionalización aterrizados a las realidades, necesidades, expectativas y particularidades de cada una de las 29 IES beneficiarias del proyecto, además de una notable sensibilización sobre la importancia de la internacionalización, su carácter transversal y el rol de todos los estamentos de una IES en su implementación.
La Universidad de Ciencias Aplicadas y Ambientales U.D.C.A, como líder del grupo gestor de las fases I y II de este proyecto de construcción de procesos propios, realistas y sustentables a partir de propia su trayectoria institucional en internacionalización, presentó su experiencia, en el Congreso de las Américas sobre Educación Superior CAEI, en Río de Janeiro, el 27 de abril de 2012.

\section{La internacionalización: factor fundamental de calidad y de fortalecimiento institucional}

La internacionalización de la Educación Superior es un fenómeno que inicia con fuerza en Colombia y, en general, en los países de América Latina y el Caribe, en los años 90 y que toma mayor reconocimiento en 1998, una vez que la Conferencia Mundial de Educación Superior de la UNESCO, explicita la dimensión internacional en su declaración mundial, lo cual, se corrobora y se potencia en la declaración de la Segunda Conferencia, casi diez años después, en 2009, en París.

A dicha dimensión internacional se le identifica como un elemento intrínseco de la calidad, que redunda en el establecimiento de redes de conocimiento, en la vinculación de comunidades académicas, en el uso de las tecnologías de la información y de la comunicación y en la promoción de lazos de cooperación, fundamentada en la ayuda mutua y la solidaridad.

En términos generales, la internacionalización tiene unos objetivos generales, que conducen a beneficios para las sociedades:

- Promover la calidad de la Educación Superior

- Impulsar la movilidad de los miembros de las comunidades académicas y su inserción en un mundo globalizado, muy especialmente, en el caso de los egresados

- Fomentar la competitividad de las IES de un país o región

- Facilitar la transferencia del conocimiento y la innovación

- Responder a la globalización sin perder en el intento, $y$, por el contrario, convirtiendo en oportunidades las amenazas que conlleva 
En la actualidad, la internacionalización de la educación superior plantea un nuevo reto y es el de la compatibilidad y comparabilidad de los sistemas nacionales de educación superior, ejercicio de convergencia basado en principios de calidad y de confianza; para alcanzar estas dos condiciones, la internacionalización de la Educación Superior debe proponer un trabajo institucional en doble vía: "por una parte, la internacionalización constituye un objetivo y un proceso que se manifiesta especialmente hacia el interior de la institución $y$, por otra, la internacionalización constituye una estrategia para difundir su oferta educativa y de investigación en un espacio supranacional" (SEBASTIÁN, Jesús. 2004. Cooperación e internacionalización de las universidades. Editorial Biblos, España. p.15). De esta manera, y ante las exigencias de la globalización respecto a la formación de profesionales comprometidos con su entorno y con las realidades de las sociedades, la internacionalización en las IES se constituye en un complejo reto que obliga a una reflexión institucional, promovida desde sus directivas, que posibilite una cultura de la internacionalización que permita proyectar sus actividades y funciones sustantivas.

La internacionalización, como proceso, requiere de unos lineamientos claros, que resulten coherentes y pertinentes con la identidad y naturaleza de las instituciones, fundamentado en una política de carácter transversal y en la definición de una estrategia consolidada en un plan de acción, como única alternativa para definir caminos y medios, según los intereses y las condiciones de cada institución.

El desarrollo de una internacionalización ajustada a las realidades de la institución y articulada con los demás procesos institucionales, contribuye al fortalecimiento institucional como un todo y a alcanzar logros duraderos en los objetivos de las tres funciones sustantivas -docencia, investigación y proyección social. Adicionalmente, en el espacio supranacional, se accede a la posibilidad de abrir nuevos espacios, de articularse al contexto mundial y de ampliar las oportunidades para los miembros de su comunidad educativa.

\section{Antecedentes y evolución de un trabajo de construcción} nacional

Un estudio realizado en 2007 por la RCI y liderado por la U.D.C.A y la Universidad de La Sabana, sobre el estado del arte de la internacionalización en Colombia, evidenció lo que se anticipaba sobre la heterogeneidad y la asimetría en el desarrollo de este proceso en las IES del país. Con una participación superior al 50\% de las instituciones, el estudio dejó entrever las brechas existentes entre las IES colombianas, según su localización (capital-región), su tamaño y su tipo (universidades/instituciones universitarias-Instituciones Téc- nicas y Tecnológicas) y la diversidad en la interpretación sobre lo que conlleva un proceso de internacionalización. Vale la pena mencionar que los resultados de este estudio, se constituyeron en la base para la construcción de los planes de acción del Ministerio de Educación Nacional y de la RCI y condujeron a un mayor trabajo conjunto entre ambas organizaciones a partir de la fecha, a lo cual, se sumaría ASCUN, como socio natural de la $\mathrm{RCI}$.

En el 2008, ante la necesidad identificada de brindar herramientas a las instituciones para iniciar o consolidar sus procesos de internacionalización, se realizaron dos talleres de capacitación, donde se socializó una serie de experiencias exitosas, en diferentes ámbitos de la internacionalización. Ello se planteó como una actividad inicial de una dinámica de construcción de competencias y conocimientos institucionales en internacionalización y favoreció el trabajo en red a nivel regional e intrarregional.

En concordancia, en el 2009, se inicia la implementación de la Acción para el Fortalecimiento del Proceso de Internacionalización de las IES Colombianas, que se basa en la necesidad que tienen las IES de hallar modelos propios para emprender una cultura internacional y llevar a la práctica una estrategia de internacionalización, fundamentada en la acción conjunta al interior de las instituciones y en una verdadera política institucional, como pilar del proceso. Esta iniciativa estaba orientada a la construcción de capacidades institucionales para la internacionalización a partir dos componentes: el primero, la realización de una serie de actividades de capacitación dirigidas a todos los miembros de las comunidades académicas de las IES del país y, el segundo, un programa de acompañamiento para la creación de políticas y de planes específicos a la medida de las IES seleccionadas, tras una convocatoria abierta.

Ante los resultados obtenidos y la identificación de un número creciente de IES interesadas en iniciar o avanzar en sus procesos de internacionalización, en el 2010, se implementó una segunda fase de esta iniciativa, que incluyó entre las beneficiarias a las instituciones técnicas y tecnológicas.

Al finalizar dos fases, el balance presentaba resultados contundentes y tangibles: 29 IES contaban con una política institucional y un plan de internacionalización, construidos con la participación de sus directivas y comunidades universitarias; la RCI creció en número de miembros y en participación de las regiones; se generó un espacio nacional de internacionalización con proyección en América Latina y el Caribe que ha agrupado en un solo espacio anual los esfuerzos y compromiso de diferentes actores (RCI, ASCUN, MEN, Cancillería, ICETEX) en torno a un tema común; se habían realizado 2 Ruedas de Alianzas con IES de América Latina 
y el Caribe en colaboración con la Cancillería, y se dio una indudable consolidación del tema de la internacionalización de la Educación Superior, como aspecto ineludible, en el marco de los procesos de calidad y de fortalecimiento institucional. Otros resultados, menos palpables, pero tanto o más importantes, de este proyecto en sus dos fases iniciales, fueron la generación de una "democratización" de la internacionalización; la reafirmación que no existe un modelo ideal para la implementación del proceso sino que puede haber tantos esquemas como instituciones y que los paradigmas importados solo funcionan si son adaptados a las realidades del entorno nacional y local y, finalmente, la identificación de las falencias y las debilidades del contexto y otras relacionadas con la gestión de las instituciones, que obstaculizan una eficiente inserción en la dinámica de la internacionalización.

A partir de 2011, el proceso de fortalecimiento nacional se ha concentrado en el Programa de Acompañamiento, a partir de un nuevo diseño, que busca aprovechar la experiencia de las universidades colombianas que cuentan con acreditación institucional de alta calidad y favorecer un ambiente de colaboración de aquellas con otras IES de menor desarrollo relativo, con la finalidad de cerrar brechas y promover la creación de lazos interinstitucionales e incrementar el trabajo en red, especialmente en las regiones.

Hacia la construcción de modelos propios de internacionalización

El programa de acompañamiento implementado en 2009 y 2010, surgió en respuesta a la necesidad de contar con una asesoría experta para el fortalecimiento del proceso de internacionalización, teniendo en cuenta, que es común que las iniciativas que se derivan de este objetivo obedecen a acciones aisladas y son producto de las circunstancias o de las voluntades personales de miembros de las instituciones.

A partir de su propia experiencia institucional y del intercambio de información frecuente en el seno de la $\mathrm{RCI}$ sobre el ejercicio de la internacionalización en otras IES, la U.D.C.A lideró el grupo gestor de la Acción para el Fortalecimiento de la Internacionalización en las IES Colombianas, que incluía entre sus componentes el Programa de Acompañamiento. Para ello, se tuvieron en cuenta cinco premisas fundamentales, a fin de generar la construcción y posterior consolidación de modelos PES (Pertinentes-Eficientes-Sostenibles):

1. No existe un modelo único para la gestión de la internacionalización en una IES, toda iniciativa debe estar acorde a la naturaleza e identidad de cada institución $\mathrm{y}$ responder a sus necesidades y proyecciones, aprovechando fortalezas, potenciando capacidades y adaptando debilidades, de manera positiva, es decir, debe ser pertinente y realista.

2. La internacionalización no es asunto exclusivo de una unidad o dependencia, es un proceso que se construye con la concurrencia de todos o casi todos los actores institucionales, es transversal y debería ser intrínseca a la gestión de todos los funcionarios, académicos y administrativos, en el marco de la política y el plan de desarrollo institucionales.

3. La construcción de un proceso de internacionalización, se debe sustentar en una autoevaluación y una dinámica de autoconocimiento institucional, que permita sustentar la definición de lineamientos y de orientaciones en ese ámbito, que correspondan coherentemente a la institución y a las realidades propias y de su entorno.

4. Es indispensable que se garantice la sostenibilidad del proceso de internacionalización, así como su coherencia y su contribución al logro de las metas institucionales, con la generación de una política de carácter institucional que alinee todos los esfuerzos bajo un mismo derrotero y garantice la voluntad y el compromiso de la institución en su adopción e implementación.

5. La internacionalización para que resulte eficiente y tenga un objetivo establecido, se debería considerar como un medio para el logro de los objetivos institucionales y no como un fin de su gestión.

A partir de este trabajo, se definieron dos fases centrales del programa:

Fase I: Acondicionamiento. Comprende: i) La sensibilización, respecto al tema de los miembros de la comunidad institucional y la socialización de temáticas y de las etapas propias del proceso de construcción institucional; ii) La autoevaluación y diagnóstico de las condiciones de la institución, tanto en su proceso de internacionalización per sé (si había tal) como de las realidades institucionales frente a los objetivos y los mecanismos de la internacionalización y iii) la definición por parte de la institución, de manera que se asegurara la correspondencia con la identidad, la naturaleza y las proyecciones institucionales.

Fase II: Asimilación y Apropiación. Con base en las recomendaciones y los aspectos resultantes de la Fase I, se formuló una propuesta delineamientos depolítica institucional de internacionalización y la posterior construcción de un plan de internacionalización de la institución. Como parte crucial de este trabajo, se llevó a cabo una evaluación de la unidad de relaciones internacionales, de fondo y de forma, con la generación de sugerencias para su reorganización y su fortalecimiento. En el plan de acción, se incluyeron metas e indicadores de gestión, así como el compromiso de implementar una estrategia de autoevaluación de los avances del proceso de internacionalización, con el objeto 
de introducir mejoras y hacer seguimiento a su desarrollo, así como una retroalimentación permanente.

El Programa de Acompañamiento para la generación de procesos viables de internacionalización, enfrentó obstáculos importantes para su implementación y para la consecución de los productos, que suponía su desarrollo. Algunos de los más frecuentes fueron:

- Las instituciones tenían una noción generalizada de la internacionalización.

- Existencia de una creencia que la internacionalización, para que sea eficiente, debía contemplar todas las manifestaciones, mecanismos y actividades posibles, sin tomar en cuenta las condiciones y las capacidades de las instituciones para su implementación.

- Las IES en sus procesos de internacionalización tenían, como únicos referentes, los modelos de las universidades más desarrolladas en este sentido.

- La internacionalización, se percibía como una acción limitada a la movilidad académica y las unidades de relaciones internacionales, como agencias que apoyaban los viajes hacia el exterior.

- Se consideraba que las acciones de internacionalización eran responsabilidad exclusiva de una sola unidad al interior de las IES.

- Suponían que la implementación de un proceso de internacionalización era garantía inmediata en la consecución de recursos de financiación no rembolsable.

- Las instituciones percibían la internacionalización como una mera estrategia de venta de servicios en el exterior.

- Noción de la internacionalización, como un fin de la gestión institucional y no como un medio para la consecución de objetivos.

\section{Diversidad de modelos, diversidad de instituciones}

Después de dos años de trabajo conjunto para la construcción de capacidades institucionales y la generación de modelos de internacionalización a la medida de las instituciones, se identificaron diversos modelos y tendencias de internacionalización, de acuerdo con tres categorías: Objeto, Relacionamiento y Administración.

Según el objeto de la internacionalización. El "QUÉ" entiende la institución como internacionalización a partir de su identidad y su naturaleza; el "QUÉ" se pretende desarrollar en el corto, mediano y largo plazo y el "PARA QUÉ" se acomete los esfuerzos y se plantean los objetivos. Asumir todos los ámbitos de la internacionalización (movilidad, investigación, currículo, internacionalización en casa, exportación de servicios, gestión de recursos y otros) $\mathrm{u}$ orientar esfuerzos hacia las áreas y mecanismos, que resultan estratégicos para la institución y para los que existen las condiciones necesarias de implementación. Como resultado, se generan modalidades distintas acopladas a la realidad y a las finalidades establecidas por cada institución, algunas de las cuales -las más representativas-, se enuncian a continuación:

1. Internacionalización basada en la función de Docencia

2. Internacionalización basada en la función de Investigación

3. Internacionalización basada en la función de Extensión

4. Internacionalización basada en la Exportación de Servicios

5. Internacionalización basada en la Gestión de Recursos

Según la política de Relacionamiento. Una vez sentadas las bases de lo que se entiende por internacionalización, sus implicaciones y connotaciones, sus alcances y objetivos institucionales, es necesario definir las prioridades de la institución, en lo que se refiere al establecimiento de sus lazos y vínculos de colaboración, el "CON QUIÉN" y las motivaciones de la decisión ("POR QUÉ"). En este sentido, se identifican varias modalidades diferentes, que no son excluyentes, pero que sí trazan un sendero que facilita la gestión de la internacionalización y le otorga a cada institución un sello, a la vez, que un derrotero claro:

1. Por prioridad geográfica

2. Por tipo de institución

3. Por tipo de relación

4. Por tipo de acuerdo

Según la administración del proceso de internacionalización. Como factor fundamental en la gestión eficiente del modelo de internacionalización y sus productos y elementos es imprescindible la definición de "QUIÉN" liderará el proceso al interior de la institución y coordinará su asimilación y apropiación para que, en efecto, se trate de un esfuerzo institucional, transversal y constructivo. En virtud de lo anterior, las IES definen diferentes modelos de administración del proceso de internacionalización, resultando los más frecuentes los siguientes:

1. Por tipo de estructura

2. Por tipo de gestión

3. Por ubicación el en organigrama

\section{Reflexiones finales}

Producto del trabajo en la generación de proceso de internacionalización en IES de todo tipo e índole en Colombia, se indicaron las siguientes consideraciones

- Una internacionalización eficiente debe servir a los objetivos institucionales $\mathrm{y}$, en consecuencia, debe ser flexible para adaptarse a las necesidades de la institución, sin perder el norte fijado en la política de internacionalización.

- Una institución solo debe implementar aquellos mecanismos o estrategias que se adapten a su política 
institucional y a sus condiciones y capacidades presentes y proyectadas. No es aconsejable asumir nuevos programas o proyectos en función únicamente de las tendencias internacionales, pues ello puede ir en detrimento de la sostenibilidad del proceso y del logro de objetivos planteados en los planes de acción institucionales.

- La internacionalización no se puede convertir en un asunto de "moda" y sus directrices e implementación han de estar arraigados en el proyecto educativo institucional, como un instrumento de generación de oportunidades, condiciones y escenarios para el cumplimiento de objetivos.

- Una institución podría generar sus propias estrategias y mecanismos, ya que está en permanente construcción y evolución. Además, como se ha mencionado, al ser la internacionalización un medio para el fortalecimiento institucional, se tendría que pensar en función del cumplimiento de las metas de desarrollo de la institución y, por tanto, y en virtud de su cualidad de flexibilidad, sería casi inevitable que cada modelo contara con algunos mecanismos propios y originales o, al menos, con adaptaciones muy particulares de los esquemas originales.

- No existe un modelo único para la internacionalización.

- Probablemente, si todos los ejercicios de implantación tendieran a procesos PES (Pertinentes-Eficientes-Sostenibles), habría tantos modelos de internacionalización como IES.

Si quiere consultar el documento completo, ingrese a la Página Web de la Universidad (http://www.udca.edu.co/es/ rectoria/noticias.html) y háganos llegar sus comentarios al correo electrónico: ganzola@udca.edu.co o relint@udca. edu.co. 